\title{
Risk factors of different types of acute pain after laparoscope-assisted vaginal hysterectomy
}

\author{
Si-Jia Chen ${ }^{1, \dagger}$, Wen-Wen Du ${ }^{1, \dagger}$, Yun-Chang Mo${ }^{1}$, Jun-Lu Wang ${ }^{1,2}$, Lu-Ping Huang ${ }^{1, *}$
}

${ }^{1}$ Department of Anesthesiology of The First Affiliated Hospital of Wenzhou Medical University, Wenzhou, P. R. China ${ }^{2}$ Department of Anesthesiology, The People's Hospital of Wencheng, Wenzhou, P. R. China

*Correspondence
csj0122@wmu.edu.cn
(Lu-Ping Huang)
${ }^{\dagger}$ These authors contributed equally.

\begin{abstract}
Purpose: Previous study has shown that acute pain after laparoscope-assisted vaginal hysterectomy (LAVH), which consists of several pain components, like somatic pain, visceral pain, shoulder pain, and low back pain (LBP). Potential risk factors of postoperative pain remain unclear. This study aimed to determine risk factors for different types of acute postoperative pain and provide guidance for personalized analgesic medication programs.

Patients and methods: This was a prospective, observational cohort study, in which 287 patients undergoing LAVH between January, 2017 and September, 2018 at the First Affiliated Hospital of Wenzhou Medical University, China were included. Univariate and multivariable logistic regression analyses were used to evaluate risk factors for the different types of acute pain after LAVH.

Results: In the total cohort, visceral pain and LBP were the most common complaints of patients, followed by somatic pain and shoulder pain. Multivariable analysis revealed that postoperative nausea and vomiting, BMI and main indication for surgery were associated with acute postoperative visceral pain. Preoperative chronic pain, BMI, preoperative anxiety and the number of trocars were associated with acute postoperative LBP, while occupation, pelvic adhesions, and history of abdominal surgery were risk factors for acute postoperative somatic pain.

Conclusion: Our study highlights that acute postoperative visceral pain and LBP are main components of pain after LAVH, and different types of acute pain after LAVH are correlated with preoperative, intraoperative and postoperative factors. Acute postoperative pain has unique risk factors according to the component type.
\end{abstract}

\section{Keywords}

Risk factors; Visceral pain; Low back pain; Somatic pain; Laparoscope-assisted vaginal hysterectomy

\section{Introduction}

According to a US national survey, $86 \%$ of patients experienced pain after surgery, and a high proportion of $75 \%$ of those patients experienced moderate to severe pain during the immediate postsurgical period [1]. Although local anesthetics, opiates, or cyclo-oxygenase (COX) inhibitors that are administered intraoperatively and immediately after operation achieve successful pain control, acute postoperative pain remains a major clinical problem and the most frequent complaint of patients. Pain sensitivity varies according to individual difference, and pain ratings of identical noxious stimuli may span the entire visual analogue scale (VAS) [2, 3]; moreover, acute postoperative pain attained high prevalence in Chinese patients undergoing surgery.

Compared with abdominal hysterectomy, laparoscopic hysterectomy has many advantages, such as small abdominal incision, fewer complications, shorter hospital stay, faster re- covery of normal life and work, and good acceptance by patients [4, 5]. Laparoscopic surgery was introduced as a minimally invasive operation, but studies showed that it may be associated with severe pain and strong need for analgesia immediately after surgery [6]. The average of postoperative pain after hysterectomy under general anesthesia was approximately 3-6 on numerical rating scale (NRS) ranging from 0 to 10 [7-9], and clinicians should consider this fact during case management.

Despite increasing understanding of acute postoperative pain in terms of mechanisms, treatment options, and evidencebased management guidelines [10], the predictors of pain in routine clinical practice remain unclear. Reports indicated several risk factors for acute postoperative pain such as preoperative pain, type of surgery, age, body mass index (BMI), and psychosocial factors [11-14], but to the best of our knowledge, no study has focused on risk factors for different types of acute postoperative pain after laparoscope-assisted 
TA B L E 1. Demographic and patient characteristics on baseline before surgery for laparoscope-assisted vaginal hysterectomy

\begin{tabular}{|c|c|c|c|}
\hline \multirow[t]{2}{*}{ Variables } & \multirow{2}{*}{$\begin{array}{c}\text { Complete cohort } \\
\text { n }=287\end{array}$} & \multirow{2}{*}{$\begin{array}{c}\text { Excluded or lost } \\
\qquad \mathrm{n}=120\end{array}$} & \multirow[t]{2}{*}{$P$ value } \\
\hline & & & \\
\hline Age (year), Mean \pm SD & $49.20 \pm 6.79$ & $49.64 \pm 6.54$ & 0.54 \\
\hline Medical Insurance Type, n (\%) & & & 0.47 \\
\hline No & $23(8)$ & $10(8.33)$ & \\
\hline New Type Of Rural Cooperative & $195(67.9)$ & $73(60.83)$ & \\
\hline Urban Residents & $10(3.5)$ & $7(5.83)$ & \\
\hline Urban Workers & $59(20.6)$ & $30(25)$ & \\
\hline Education, n (\%) & & & 0.53 \\
\hline Illiteracy & $71(24.7)$ & $35(29.2)$ & \\
\hline Primary School & $123(42.9)$ & $42(35)$ & \\
\hline Middle And High School & $75(26.1)$ & $35(29.17)$ & \\
\hline College And Higher & $18(6.3)$ & $8(6.67)$ & \\
\hline Occupation, n (\%) & & & 0.06 \\
\hline No & $170(59.2)$ & $59(49.2)$ & \\
\hline Yes & $117(40.8)$ & $61(50.8)$ & \\
\hline Hypertension, n (\%) & & & 0.64 \\
\hline No & $226(78.7)$ & $97(80.8)$ & \\
\hline Yes & $61(21.3)$ & $23(19.2)$ & \\
\hline Diabetes, n (\%) & & & 0.28 \\
\hline No & $270(94.1)$ & $116(96.7)$ & \\
\hline Yes & $17(5.9)$ & $4(3.3)$ & \\
\hline A History Of Abdominal Surger & & & 0.17 \\
\hline No & $213(74.2)$ & $81(67.5)$ & \\
\hline Yes & $74(25.8)$ & $74(32.5)$ & \\
\hline Cesarean Section, n (\%) & & & 0.12 \\
\hline No & $253(88.2)$ & $112(93.3)$ & \\
\hline Yes & $34(11.8)$ & $8(6.7)$ & \\
\hline Dysmenorrhea, n (\%) & & & 0.08 \\
\hline No & $187(65.2)$ & $187(74.2)$ & \\
\hline Yes & $100(34.8)$ & $31(25.8)$ & \\
\hline BMI $\left(\mathrm{kg} / \mathrm{m}^{2}\right)$, Mean \pm SD & $23.67 \pm 2.96$ & $23.60 \pm 2.37$ & 0.85 \\
\hline Main indication for surgery, $\mathbf{n}$ & & & 0.10 \\
\hline Physical Findings & $179(62.4)$ & $60(50)$ & \\
\hline Descensus Uteri & $15(5.2)$ & $6(5)$ & \\
\hline Changes In Menstruation & $68(23.7)$ & $42(35)$ & \\
\hline Dysmenorrhea & $25(8.7)$ & $12(10)$ & \\
\hline Preoperative Chronic Pain, n (\% & & & 0.13 \\
\hline No & $201(70)$ & $93(77.5)$ & \\
\hline Yes & $86(30)$ & $27(22.5)$ & \\
\hline Exercise Habit, n (\%) & & & 0.80 \\
\hline No & $219(76.3)$ & $93(77.5)$ & \\
\hline Yes & $68(23.7)$ & $27(22.5)$ & \\
\hline The Level Of Anxiety, n (\%) & & & 0.14 \\
\hline Mild & $213(74.2)$ & $99(82.5)$ & \\
\hline Moderate & $44(15.3)$ & $10(8.3)$ & \\
\hline Severe & $30(10.5)$ & $11(9.2)$ & \\
\hline
\end{tabular}

Data are presented as $n(\%)$ or mean \pm standard deviation.

Statistics are t test, sank sum test or chi-square test wherever appropriate.

vaginal hysterectomy (LAVH).

Based on these facts, we performed the present prospective, observational cohort study to support that acute pain after LAVH consists of several pain components: somatic pain, visceral pain, shoulder pain and low back pain (LBP) $[15,16]$; the hypothesis of the study is that there are differences in risk factors of acute pain according to symptom location.

\section{Methods}


TA B L E 2. Incidence of different types of postoperative pain

\begin{tabular}{|lcccc|} 
Type of pain & NO, $\mathrm{n}(\%)$ & Mild, $\mathrm{n}(\%)$ & Moderate, $\mathrm{n}(\%)$ & Severe, $\mathrm{n}(\%)$ \\
\hline Visceral pain & $33(11.50)$ & $129(44.95)$ & $69(24.04)$ & $56(19.51)$ \\
\hline Somatic pain & $83(28.92)$ & $140(48.78)$ & $58(20.21)$ & $6(2.10)$ \\
\hline Low back pain & $67(23.34)$ & $112(39.02)$ & $63(21.95)$ & $45(15.68)$ \\
Shoulder pain & $243(84.67)$ & $28(9.76)$ & $9(3.14)$ & $7(2.44)$ \\
\hline
\end{tabular}

Values are presented as $n(\%)$.

Patients screened for eligibility preoperatively $n=407$

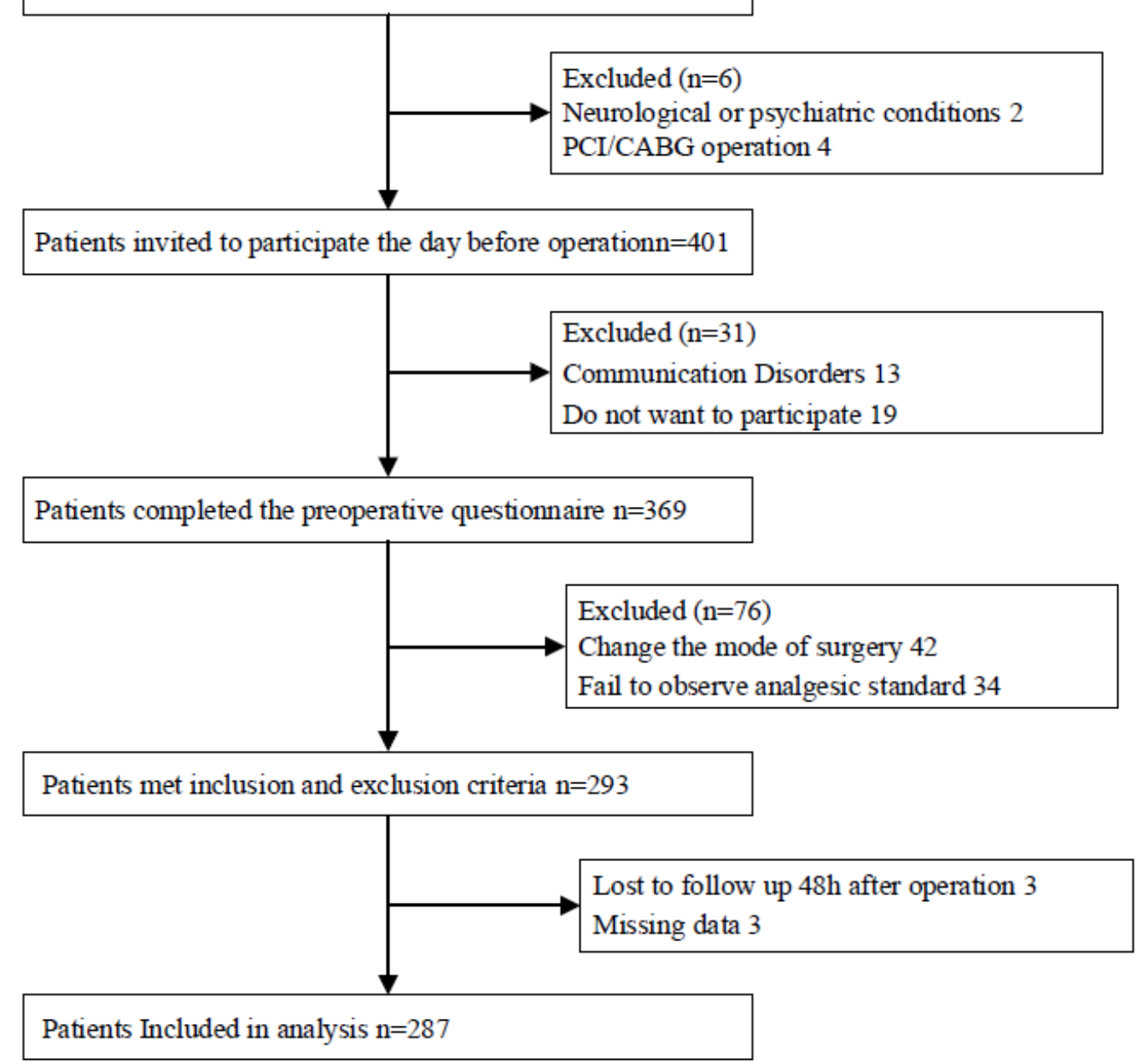

\section{F I G U RE 1. Flowchart of the patients.}

\subsection{Study design and participant recruitment}

This prospective, observational cohort study was approved by the Ethics Committee (2019-030) in Clinical Research of the First Affiliated Hospital of Wenzhou Medical University and was registered at China Clinical Trial Registry: ChiCTR-ROC17013036. Patients scheduled for LAVH at the Gynecology Department at First Affiliated Hospital of Wenzhou Medical University between January, 2017 and October, 2018 were enrolled in the study on the day before surgery. Written informed consent was obtained from every participant by preoperative interview on the same day; subsequently, data of patient characteristics were collected. Data of futher information about the surgery, anesthesia and pain were collected by researcher on the day of operation and $24 \mathrm{~h}, 48 \mathrm{~h}$ after operation. Data of all patients who completed the entire study were submitted to analysis.

From January, 2017 to October, 2018, indices of potential acute postoperative pain were recorded in a single database. Patients scheduled for LAVH under general anesthesia were recruited; at the time of registration, eligible patients were $>18$ years-old. Those with serious psychological disorder and other significant cardiovascular, respiratory, hepatic and 
TA B L E 3. Univariate analysis of risk factors of acute postoperative visceral pain No Pain Or Mild Pain, n (\%) Moderate To Severe Pain, n (\%) P value OR (95\% CI)

Preoperative variable

Age

$\leq 45$

$45-55$

$42(25.9)$

$93(57.4)$

$>55$

$27(16.7)$

$38(30.4)$

Medical Insurance Type

No 14 (8.6)

New Type Of Rural Cooperative 112 (69.1)

Urban Residents

Urban Workers

8 (4.9)

Education

Illiteracy

$28(17.3)$

Primary School

$41(25.3)$

Middle And High School

College And Higher

$73(45.1)$

Occupation

No

$42(25.9)$

$6(3.7)$

$73(58.4)$

$14(11.2)$

0.36

Yes

$99(61.1)$

$63(38.9)$

Hypertension

No

$122(75.3)$

Yes

40 (24.7)

Diabetes

No

$149(92)$

Yes

$13(8)$

A History Of Abdominal Surgery

No

$118(72.8)$

Yes

$44(27.2)$

Cesarean Section

No

$145(89.5)$

Yes

$17(10.5)$

Parity

$\leq$ One

Two

$53(32.7)$

$>$ Two

$75(46.3)$

$34(21)$

Abortion

$\leq$ One

$62(38.3)$

Two

$44(27.2)$

$>$ Two

$56(34.6)$

Dysmenorrhea

No

$110(54.6)$

$9(7.2)$

$83(66.4)$

$2(1.6)$

$31(24.8)$

$0.60 \quad 0.87(0.51-1.48)$

$0.16 \quad 0.57(0.26-1.25)$

0.20

$0.75 \quad 1.15(0.48-2.79)$

$0.29 \quad 0.39(0.07-2.26)$

$0.28 \quad 1.72(0.65-4.59)$

$30(24)$

$50(40)$

$33(26.4)$

$12(9.6)$

0.22

$71(56.8)$

$54(43.2)$

$0.83 \quad 0.94(0.52-1.69)$

$0.83 \quad 1.07(0.56-2.07)$

$0.07 \quad 2.73(0.92-8.11)$

0.46

$104(83.2)$

$21(16.8)$

$121(96.8)$

4 (3.2)

$0.46 \quad 1.2(0.74-1.92)$

0.10

$0.11 \quad 0.62(0.34-1.11)$

0.08

Yes $52(45.4)$

BMI

$<24$

$\geq 24$

82 (67.9)

$95(76)$

$30(24)$

$0.10 \quad 0.38(0.12-1.19)$ 0.54

$0.54 \quad 0.85(0.5-1.45)$

$108(86.4)$

17 (13.6)

$0.42 \quad 1.34(0.66-2.75)$

$40(32)$

$61(48.8)$

$24(19.2)$

0.90

Main indication for surgery

Physical Findings

$80(32.1)$

Descensus Uteri

$98(60.5)$

$14(8.6)$

Changes In Menstruation $34(21)$

Dysmenorrhea

$16(9.9)$

49 (39.2)

$41(32.8)$

35 (28)

$0.78 \quad 1.08(0.63-1.83)$

$0.84 \quad 0.94(0.48-1.82)$

0.42

Preoperative Chronic Pain

No

Yes

$116(71.6)$

$46(28.4)$

77 (61.6)

48 (38.4)

$0.57 \quad 1.18(0.67-2.08)$

$0.42 \quad 0.79(0.45-1.39)$

0.27

$0.27 \quad 1.32(0.81-2.15)$

79 (63.2)

$46(36.8)$

0.03

$81(64.8)$

$1(0.8)$

$34(27.2)$

$9(7.2)$

$0.03 \quad 0.6(0.37-0.96)$

\section{Exercise Habit}

No

125 (77.2)

$37(22.8)$

85 (68)

$40(32)$

0.01

$0.02 \quad 0.09(0.01-0.67)$

$0.50 \quad 1.21(0.69-2.12)$

$0.390 .68(0.29-1.62)$

Yes

117 (72.2)

$94(75.2)$

$31(24.8)$

0.51

The Level Of Anxiety

Mild

$26(16)$

$96(76.8)$

Moderate

$19(11.7)$

$18(14.4)$

$11(8.8)$

$0.51 \quad 1.18(0.71-1.97)$

0.70

$0.70 \quad 1.11(0.64-1.93)$

0.63

Severe

$0.61 \quad 0.84(0.44-1.63)$

$0.39 \quad 0.71(0.32-1.55)$ 
TA B L E 3. Continued

\begin{tabular}{|c|c|c|c|c|}
\hline & \multicolumn{3}{|c|}{ No Pain Or Mild Pain, $n(\%)$ Moderate To Severe Pain, $n(\%) P$ value } & \multirow[t]{2}{*}{ OR $(95 \% \mathrm{CI})$} \\
\hline Pelvic Adhesions & & & 0.39 & \\
\hline No & $101(62.4)$ & $84(67.2$ & & \\
\hline Yes & $61(37.6)$ & $41(32.8$ & 0.39 & $0.81(0.5-1.32)$ \\
\hline \multicolumn{3}{|l|}{ The Number Of Trocar } & 0.74 & \\
\hline Three & $72(44.4)$ & $58(46.4$ & & \\
\hline$\geq$ Four & $90(55.6)$ & $67(53.6$ & 0.74 & $0.92(0.58-1.48)$ \\
\hline Uterine Size & & & 0.29 & \\
\hline Atrophy & $20(12.3)$ & $15(12)$ & & \\
\hline Normal & $19(11.7)$ & $7(5.6)$ & 0.20 & $0.49(0.16-1.47)$ \\
\hline First Trimester Of Pregnancy & $55(34)$ & $50(40)$ & 0.63 & $1.21(0.56-2.62)$ \\
\hline Second Trimester Of Pregnancy & $68(42)$ & $53(42.4$ & 0.92 & $1.04(0.49-2.22)$ \\
\hline \multicolumn{3}{|l|}{ Bleeding volume } & 0.7 & \\
\hline$\leq 20$ & $88(54.3)$ & $65(52)$ & & \\
\hline $20-50$ & $49(30.2)$ & $36(28.8$ & 0.98 & $0.99(0.58-1.7)$ \\
\hline$>50$ & $25(15.4)$ & $24(19.2$ & 0.43 & $1.3(0.68-2.48)$ \\
\hline \multicolumn{3}{|l|}{ Operative time } & 0.5 & \\
\hline$\leq 120 \min$ & $131(80.9)$ & $97(77.6$ & & \\
\hline$>120 \mathrm{~min}$ & $90(19.1)$ & $28(22.4$ & 0.50 & $1.22(0.69-2.17)$ \\
\hline \multicolumn{3}{|c|}{ Post-Operative Nausea And Vomiting } & 0.04 & \\
\hline No & $120(74.1)$ & $75(60)$ & & \\
\hline Only Nausea & $12(7.4)$ & $16(12.8$ & 0.06 & $2.13(0.96-4.76)$ \\
\hline Vomiting & $30(18.5)$ & $34(27.2$ & 0.04 & $1.81(1.03-3.2)$ \\
\hline \multicolumn{3}{|l|}{ Time Of Pelvic Drainage } & 0.78 & \\
\hline One Day & $128(79)$ & $96(76.8$ & & \\
\hline Two Days & $25(15.4)$ & $23(18.4$ & 0.52 & $1.23(0.66-2.29)$ \\
\hline Three Days Or More & $9(5.6)$ & $6(4.8)$ & 0.83 & $0.89(0.31-2.58)$ \\
\hline \multicolumn{3}{|l|}{ The Indwelling Catheter Time } & 0.35 & \\
\hline No or One Day & $22(13.6)$ & $24(18.5$ & & \\
\hline Two Days & $67(41.4)$ & $47(37.6$ & 0.21 & $0.64(0.32-1.28)$ \\
\hline Three Days Or More & $73(45.1)$ & $54(43.2$ & 0.26 & $0.67(0.34-1.33)$ \\
\hline \multicolumn{3}{|c|}{ Volume of pelvic drainage in $24 \mathrm{~h}$} & 0.51 & \\
\hline$\leq 50$ & $57(35.2)$ & $48(38.4$ & & \\
\hline$\overline{50}-100$ & $53(32.7)$ & $33(26.4$ & 0.31 & $0.74(0.41-1.32)$ \\
\hline$>100$ & $52(32.1)$ & $44(35.2$ & 0.99 & $1(0.58-1.75)$ \\
\hline
\end{tabular}

Values are presented as $n(\%)$;

OR, odds ratio; CI, Confidence interval;

Binary logistic regression analysis.

kidney diseases were excluded; other exclusion criteria were limitations of self-expression or language problems, pregnant mothers, and nursing mothers.

\subsection{Types of outcomes}

For development of risk assessment models, the primary outcome was incidence and intensity of acute postoperative pain, including somatic pain, visceral pain, shoulder pain, and LBP. Intra-abdominal pain (visceral pain) was defined as pain within the abdomen, which may be deep, dull, and more difficult to localize, but that may be predominantly located in the hypogastrium. Somatic pain was a superficial pain localized in the wounds that were sensitive to touch. Shoulder pain was defined as pain sensation in the shoulder(s) [9, 17]. Low back pain (LBP) was defined as pain located between the 12th rib and the inferior gluteal folds, with or without leg pain according to a previous study [18].

Pain intensity was assessed using NRS from 0 to 10 , with zero representing no pain and 10 representing worst imaginable pain [19], and categorized into two groups: no pain or mild pain, 0 to 3 ; moderate to severe pain, 4 to $10[8,20]$.

The secondary outcomes were (1) postoperative complications, such as respiratory depression, bleeding, and fever and (2) length of hospital stay, exhaust time, and time to get out of bed.

\subsection{Data collection}

Data collection and processing were completed by three anesthesiologists and four college students; preoperative data were collected and checked by two college students and entered by two other college students all who completed 1 week's training for their respective tasks. Two anesthesiologists were responsible for intraoperative anesthesia and administration of analgesia, and two university students, for intraoperative data collection. Follow-up was conducted by another anesthesiologist who was blinded to preoperative and intraoperative data, which included pain assessment, and management of postoperative pain and other complications. 
TA B L E 4. Univariate analysis of risk factors of acute postoperative low back pain No Pain Or Mild Pain N (\%) Moderate To Severe Pain N (\%) $P$ value

\section{Preoperative variable}

Age

$\leq 45$

$45-55$

$>55$

Medical Insurance Type

No

New Type Of Rural Cooperative

Urban Residents

Urban Workers

Education

Illiteracy

Primary School

Middle And High School

College And Higher

Occupation

No

Yes

\section{Hypertension}

No

Yes

Diabetes

No

Yes

A History Of Abdominal Surgery

No

Yes

\section{Cesarean Section}

No

Yes

Parity

$\leq$ One

Two

$>$ Two

Abortion

$\leq$ One

Two

$>$ Two

\section{Dysmenorrhea}

No

Yes

BMI

$<24$

$\geq 24$

Main indication for surgery

Physical Findings

Descensus Uteri

Changes In Menstruation

Dysmenorrhea

Preoperative Chronic Pain

No

Yes

\section{Exercise Habit}

No

Yes

The Level Of Anxiety

Mild

Moderate
$57(31.8)$

$100(55.9)$

$22(12.3)$

$19(10.6)$

$119(66.5)$

$6(3.4)$

$35(19.6)$

45 (25.1)

$74(41.3)$

50 (27.9)

$10(5.6)$

$110(61.5)$

$69(38.5)$

$142(79.3)$

37 (20.7)

$170(95)$

9 (5)

128 (71.5)

$51(28.5)$

$153(85.5)$

$26(14.5)$

$58(32.4)$

$86(48)$

35 (19.6)

67 (37.4)

$57(31.8)$

$55(30.7)$

$119(66.5)$

$60(33.5)$

92 (51.4)

87 (48.6)

$107(59.8)$

$7(3.9)$

$51(28.5)$

$14(7.8)$

143 (79.9)

$36(20.1)$

137 (76.5)

$42(23.5)$

$137(76.5)$

$30(16.8)$
$23(21.3)$

$66(61.1) \quad 0.09$

$19(17.6) \quad 0.06$

0.18

$4(3.7)$

$76(70.4)$

$4(3.7)$

$24(22.2)$

$0.05 \quad 3.03(0.99-9.26)$

$0.17 \quad 3.17(0.6-16.69)$

$0.05 \quad 3.26(0.98-10.78)$

0.75

$26(24.1)$

$49(45.4)$

$25(23.1)$

0.66

0.68

0.54

$1.15(0.63-2.09)$

$8(7.4)$

$0.87(0.44-1.71)$

$1.38(0.49-3.95)$

60 (55.6)

$48(44.4)$

0.33

$1.28(0.79-2.07)$

$84(77.8)$

$24(22.2)$

0.76

0.41

$100(92.6)$

$8(7.4)$

0.41

0.17

85 (78.7)

$23(21.3)$

$0.18 \quad 0.68(0.39-1.19)$

0.06

$100(92.6)$

$8(7.4)$

0.08

$0.47(0.2-1.08)$

35 (32.4)

$50(46.3)$

0.93

23 (21.3)

0.89

0.8

$0.96(0.56-1.66)$

0.56

44 (40.7)

$28(25.9)$

$36(33.3)$

0.34

0.99

0.55

$68(63.0)$

$40(37.0)$

$0.55 \quad 1.17(0.71-1.92)$

0.04

$69(63.9)$

39 (36.1)

0.04

$0.6(0.37-0.98)$

72 (66.7)

$8(7.4)$

$17(15.7)$

$11(10.2)$

0.33

$1.7(0.59-4.89)$

$0.03 \quad 0.5(0.27-0.93)$

$0.72 \quad 1.17(0.5-2.72)$

$58(53.7)$

$50(46.3)$

$<0.01 \quad 3.42(2.02-5.79)$

0.91

$82(75.9)$

$26(24.1)$

$0.91 \quad 1.03(0.59-1.81)$

$76(70.4)$

$14(13)$
0.03

$0.63 \quad 0.84(0.42-1.68)$ 
TA B L E 4. Continued No Pain Or Mild Pain N (\%) Moderate To Severe Pain N (\%) P value OR (95\% CI)

\begin{tabular}{|c|c|c|c|c|}
\hline Severe & $12(6.7)$ & $18(16.7)$ & 0.01 & $2.7(1.24-5.91)$ \\
\hline \multicolumn{5}{|l|}{ Intraoperative pain } \\
\hline \multicolumn{3}{|l|}{ Pelvic Adhesions } & \multicolumn{2}{|l|}{0.26} \\
\hline No & $111(62)$ & $74(68.5)$ & & \\
\hline Yes & $68(38)$ & $34(31.5)$ & 0.27 & $0.75(0.45-1.24)$ \\
\hline \multicolumn{3}{|l|}{ The Number Of Trocar } & \multicolumn{2}{|l|}{0.03} \\
\hline Three & $72(40.2)$ & $58(53.7)$ & & \\
\hline$\geq$ Four & $107(59.8)$ & $50(46.3)$ & 0.03 & $0.58(0.36-0.94)$ \\
\hline \multicolumn{3}{|l|}{ Uterine Size } & \multicolumn{2}{|l|}{0.9} \\
\hline Atrophy & $20(11.2)$ & $15(13.9)$ & & \\
\hline Normal & $17(9.5)$ & $9(8.3)$ & 0.52 & $0.71(0.25-2.02)$ \\
\hline First Trimester Of Pregnancy & $67(37.4)$ & $38(35.2)$ & 0.48 & $0.76(0.35-1.65)$ \\
\hline Second Trimester Of Pregnancy & $75(41.9)$ & $46(42.6)$ & 0.61 & $0.82(0.38-1.75)$ \\
\hline \multicolumn{3}{|l|}{ Bleeding volume } & \multicolumn{2}{|l|}{0.05} \\
\hline$\leq 20$ & $101(56.4)$ & $52(48.1)$ & & \\
\hline $20-50$ & $44(24.6)$ & $41(38)$ & 0.03 & $1.81(1.05-3.11)$ \\
\hline$>50$ & 34 (19) & $15(13.9)$ & 0.66 & $0.86(0.43-1.71)$ \\
\hline \multicolumn{3}{|l|}{ Operative time } & \multicolumn{2}{|l|}{0.72} \\
\hline$\leq 120 \mathrm{~min}$ & $141(78.8)$ & $87(80.6)$ & & \\
\hline$>120 \mathrm{~min}$ & $38(21.2)$ & $21(19.4)$ & 0.72 & $0.90(0.49-1.63)$ \\
\hline \multicolumn{5}{|l|}{ Postoperative pain } \\
\hline Post-Operative Nausea And Vo & & & \multicolumn{2}{|l|}{0.5} \\
\hline No & $118(65.9)$ & $77(71.3)$ & & \\
\hline Only Nausea & $20(11.2)$ & $8(7.4)$ & 0.27 & $0.61(0.26-1.46)$ \\
\hline Vomiting & $41(22.9)$ & $23(21.3)$ & 0.61 & $0.86(0.48-1.54)$ \\
\hline \multicolumn{3}{|l|}{ Time Of Pelvic Drainage } & \multicolumn{2}{|l|}{0.94} \\
\hline One Day & $139(77.7)$ & $85(78.7)$ & & \\
\hline Two Days & $30(16.8)$ & $18(16.7)$ & 0.95 & $0.98(0.52-1.87)$ \\
\hline Three Days Or More & $10(5.6)$ & $5(4.6)$ & 0.72 & $0.82(0.27-2.47)$ \\
\hline \multicolumn{3}{|l|}{ The Indwelling Catheter Time } & \multicolumn{2}{|l|}{0.09} \\
\hline NO or One Day & $35(19.6)$ & $11(10.2)$ & & \\
\hline Two Days & $70(39.1)$ & $44(40.7)$ & 0.08 & $2(0.92-4.34)$ \\
\hline Three Days Or More & $74(41.3)$ & $53(49.1)$ & 0.04 & $2.28(1.06-4.89)$ \\
\hline \multicolumn{3}{|l|}{ Volume of pelvic drainage in $24 \mathrm{~h}$} & \multicolumn{2}{|l|}{0.44} \\
\hline$\leq 50$ & $65(36.3)$ & $40(37)$ & & \\
\hline $50-100$ & $58(32.4)$ & $28(25.9)$ & 0.43 & $0.78(0.43-1.43)$ \\
\hline$>100$ & $56(31.3)$ & $40(37)$ & 0.61 & $1.16(0.66-2.04)$ \\
\hline
\end{tabular}

Values are presented as $n(\%)$;

OR, odds ratio; CI, Confidence interval;

Binary logistic regression analysis.

Variables as potential risk factors for acute postoperative pain were identified based on available literature. Preoperative variables were age, BMI, education, occupation, exercise habit, individual disease history, obstetrical history, previous surgical history, history of preoperative chronic pain, type of medical insurance, main indication for surgery, and anxiety level. Data of patient's characteristics were obtained by interview on the day before surgery.

Patient's age, height and weight, occupation, vital signs, obstetrical history, individual disease history, and surgical history were obtained by review of the medical record, and the data were verified for accuracy by staff who conducted preoperative interview. BMI was categorized dichotomously with cutoff at 24 [21, 22]. Subjects were classified by dichotomous variable according to previous major surgery with or without abdominal surgery.

In addition, history of pre-existing pain was assessed, which included aspects of its location, duration, and intensity. Patients who experienced pain for a period of $>3$ months at $\geq$ 15 days per month with intensity of $\geq 2$ on an 11-point NRS by preoperative interview were considered to have pre-existing chronic pain [23]. In the questionnaire, preoperative pain was classified according to the location of pain such as the head, cervical vertebra, waist, back, chest, abdomen, and limbs [24].

\subsection{Anesthesia, surgical procedures and analgesia}

In all patients, similar regimens of general anesthesia and prophylactic analgesia were administered without any premedication on the day of surgery. Patients were monitored with standard procedure by bispectral index (BIS) for depth of anesthesia. General anesthesia was induced with a combination of sulfentanyl $\left(0.4 \mu \mathrm{g} \cdot \mathrm{kg}^{-1}\right)$ and propofol $\left(2-3 \mathrm{mg} \cdot \mathrm{kg}^{-1}\right)$. Next, 
TA B L E 5. Univariate analysis of risk factors of acute postoperative somatic pain No Pain Or Mild Pain N (\%) Moderate To Severe Pain N (\%) P value OR (95\% CI)

\title{
Preoperative variable
}

Age

$\leq 45$

$45-55$

$>55$

\section{Nain}

Medical Insurance Type

No

New Type Of Rural Cooperative

Urban Residents

Urban Workers

\section{Education}

Illiteracy

Primary School

Middle And High School

College And Higher

Occupation

No

Yes

\section{Hypertension}

No

Yes

Diabetes

No

Yes

A History Of Abdominal Surgery

No

Yes

Cesarean Section

No

Yes

Parity

$\leq$ One

Two

$>$ Two

Abortion

$\leq$ One

Two

$>$ Two

\section{Dysmenorrhea}

No

Yes

BMI

$<24$

$\geq 24$

Main indication for surgery

Physical Findings

Descensus Uteri

Changes In Menstruation

Dysmenorrhea

\section{Preoperative Chronic Pain}

No

Yes

\section{Exercise Habit}

No

Yes

The Level Of Anxiety

\begin{tabular}{|c|c|c|c|c|}
\hline & & & 0.07 & \\
\hline & $56(25.1)$ & $24(37.5)$ & & \\
\hline & $131(58.7)$ & $35(54.7)$ & 0.13 & $0.62(0.34-1.14)$ \\
\hline & $36(16.1)$ & $5(7.8)$ & 0.04 & $0.32(0.11-0.93)$ \\
\hline & & & 0.56 & \\
\hline & $16(7.2)$ & $7(10.9)$ & & \\
\hline ative & $156(70)$ & $39(60.9)$ & 0.25 & $0.57(0.22-1.48)$ \\
\hline & $7(3.1)$ & $3(4.7)$ & 0.98 & $0.98(0.19-4.94)$ \\
\hline & $44(19.7)$ & $15(23.4)$ & 0.65 & $0.78(0.27-2.26)$ \\
\hline & & & 0.49 & \\
\hline & $52(23.3)$ & $19(29.7)$ & & \\
\hline & $97(43.5)$ & $26(40.6)$ & 0.37 & $0.73(0.37-1.45)$ \\
\hline & $58(26)$ & $17(26.6)$ & 0.57 & $0.8(0.38-1.7)$ \\
\hline & $16(7.2)$ & $2(3.1)$ & 0.18 & $0.34(0.07-1.63)$ \\
\hline & & & 0.002 & \\
\hline & $143(64.1)$ & $27(42.2)$ & & \\
\hline & $80(35.9)$ & $37(57.8)$ & 0.002 & $2.45(1.39-4.32)$ \\
\hline & & & 0.10 & \\
\hline & $171(76.7)$ & 55 (85.9) & & \\
\hline & $52(23.3)$ & $9(14.1)$ & 0.12 & $0.54(0.25-1.16)$ \\
\hline & & & 0.06 & \\
\hline & $207(92.8)$ & $63(98.4)$ & & \\
\hline & $16(7.2)$ & $1(1.6)$ & 0.13 & $0.21(0.03-1.58)$ \\
\hline urgery & & & 0.001 & \\
\hline & $176(78.9)$ & $37(57.8)$ & & \\
\hline & $47(21.1)$ & $27(42.2)$ & 0.001 & $2.73(1.51-4.94)$ \\
\hline & & & 0.02 & \\
\hline & $202(90.6)$ & $51(79.7)$ & & \\
\hline & $21(9.4)$ & $13(20.3)$ & 0.02 & $2.45(1.15-5.23)$ \\
\hline & & & 0.62 & \\
\hline & $69(30.9)$ & $24(37.5)$ & & \\
\hline & $108(48.4)$ & $28(43.8)$ & 0.36 & $0.75(0.4-1.39)$ \\
\hline & $46(20.6)$ & $12(18.8)$ & 0.47 & $0.75(0.34-1.65)$ \\
\hline & & & 0.26 & \\
\hline & $82(36.8)$ & $29(45.3)$ & & \\
\hline & $71(31.8)$ & $14(21.9)$ & 0.11 & $0.56(0.27-1.14)$ \\
\hline & $70(31.4)$ & $21(32.8)$ & 0.62 & $0.85(0.44-1.62)$ \\
\hline & & & 0.70 & \\
\hline & $144(64.6)$ & $43(67.2)$ & & \\
\hline & $79(35.4)$ & $21(32.8)$ & 0.70 & $0.89(0.49-1.61)$ \\
\hline & & & 0.80 & \\
\hline & $126(56.5)$ & $35(54.7)$ & & \\
\hline & $97(43.5)$ & $29(45.3)$ & 0.80 & $1.08(0.62-1.88)$ \\
\hline $\mathbf{y}$ & & & 0.65 & \\
\hline & $141(63.2)$ & $38(59.4)$ & & \\
\hline & $13(5.8)$ & $2(3.1)$ & 0.47 & $0.57(0.12-2.64)$ \\
\hline & $51(22.9)$ & $17(26.6)$ & 0.53 & $1.24(0.64-2.38)$ \\
\hline & $18(8.1)$ & $7(10.9)$ & 0.45 & $1.44(0.56-3.71)$ \\
\hline & & & 0.39 & \\
\hline & $159(71.3)$ & $42(65.6)$ & & \\
\hline & $64(28.7)$ & $22(34.3)$ & 0.38 & $1.3(0.72-2.35)$ \\
\hline & & & 0.46 & \\
\hline & $168(75.3)$ & $51(79.7)$ & & \\
\hline & $55(24.7)$ & $13(20.3)$ & 0.47 & $0.78(0.39-1.54)$ \\
\hline & & & 0.09 & \\
\hline
\end{tabular}

$19(29.7)$

$26(40.6)$

$7(26.6)$

$0.57 \quad 0.8(0.38-1.7)$

$0.18 \quad 0.34(0.07-1.63)$

$0.002 \quad 2.45(1.39-4.32)$

$0.12 \quad 0.54(0.25-1.16)$

$0.13 \quad 0.21(0.03-1.58)$

$0.001 \quad 2.73(1.51-4.94)$

$0.02 \quad 2.45(1.15-5.23)$

$0.36 \quad 0.75(0.4-1.39)$

$0.47 \quad 0.75(0.34-1.65)$

$0.11 \quad 0.56(0.27-1.14)$

$0.62 \quad 0.85(0.44-1.62)$

$0.70 \quad 0.89(0.49-1.61)$

$0.80 \quad 1.08(0.62-1.88)$

$0.47 \quad 0.57(0.12-2.64)$

$0.53 \quad 1.24(0.64-2.38)$

$0.45 \quad 1.44(0.56-3.71)$

$0.38 \quad 1.3(0.72-2.35)$

$\begin{array}{ll}0.47 & 0.78(0.39-1.54) \\ 0.09 & \end{array}$ 
TA B L E 5. Continued No Pain Or Mild Pain N (\%) Moderate To Severe Pain N (\%) P value $\quad$ OR (95\% CI)

\begin{tabular}{|c|c|c|c|c|}
\hline Mild & $159(71.3)$ & $54(84.4)$ & & \\
\hline Moderate & $38(17)$ & $6(9.4)$ & 0.10 & $0.46(0.19-1.16)$ \\
\hline Severe & $26(11.7)$ & $4(6.3)$ & 0.16 & $0.45(0.15-1.36)$ \\
\hline \multicolumn{2}{|l|}{ Pelvic Adhesions } & \multicolumn{3}{|c|}{$<0.001$} \\
\hline No & $156(70)$ & $29(45.3)$ & & \\
\hline Yes & $67(30)$ & $35(54.7)$ & $<0.001$ & $2.81(1.59-4.97)$ \\
\hline \multicolumn{2}{|l|}{ The Number Of Trocar } & \multicolumn{3}{|c|}{0.15} \\
\hline Three & $106(47.5)$ & $24(37.5)$ & & \\
\hline$\geq$ Four & $117(52.5)$ & $40(62.5)$ & 0.16 & $1.51(0.85-2.67)$ \\
\hline \multicolumn{2}{|l|}{$\overline{\text { Uterine Size }}$} & & \multicolumn{2}{|l|}{0.28} \\
\hline Atrophy & $26(11.7)$ & $9(14.1)$ & & \\
\hline Normal & $21(9.4)$ & $5(7.8)$ & 0.55 & $0.69(0.2-2.37)$ \\
\hline First Trimester Of Pregnancy & $76(34.1)$ & $29(45.3)$ & 0.83 & $1.1(0.46-2.63)$ \\
\hline Second Trimester Of Pregnancy & $100(44.8)$ & $21(32.8)$ & 0.27 & $0.61(0.25-1.48)$ \\
\hline \multicolumn{2}{|l|}{ Bleeding volume } & & \multicolumn{2}{|l|}{0.63} \\
\hline$\leq 20$ & $119(53.4)$ & $34(53.1)$ & & \\
\hline $20-50$ & $68(30.5)$ & $17(26.6)$ & 0.68 & $0.87(0.46-1.66)$ \\
\hline$>50$ & $36(16.1)$ & $13(20.3)$ & 0.48 & $1.3(0.63-2.68)$ \\
\hline \multicolumn{2}{|l|}{ Operative time } & & \multicolumn{2}{|l|}{0.52} \\
\hline$\leq 120 \mathrm{~min}$ & $179(80.3)$ & $49(76.6)$ & & \\
\hline$>120 \min$ & $44(19.7)$ & $15(23.4)$ & 0.52 & $1.25(0.64-2.42)$ \\
\hline \multicolumn{2}{|c|}{ Post-Operative Nausea And Vomiting } & & \multicolumn{2}{|l|}{0.97} \\
\hline No & $152(68.2)$ & $43(67.2)$ & & \\
\hline Only Nausea & $22(9.9)$ & $6(9.4)$ & 0.94 & $0.96(0.37-2.53)$ \\
\hline Vomiting & $49(22)$ & $15(23.4)$ & 0.82 & $1.08(0.55-2.12)$ \\
\hline \multicolumn{2}{|l|}{ Time Of Pelvic Drainage } & & \multicolumn{2}{|l|}{0.17} \\
\hline One Day & $170(76.2)$ & $54(84.4)$ & & \\
\hline Two Days & $42(18.8)$ & $6(9.4)$ & 0.09 & $0.45(0.18-1.12)$ \\
\hline Three Days Or More & $11(4.9)$ & $4(6.3)$ & 0.82 & $1.14(0.35-3.74)$ \\
\hline \multicolumn{2}{|l|}{ The Indwelling Catheter Time } & & \multicolumn{2}{|l|}{0.35} \\
\hline NO or One Day & $32(14.4)$ & $14(21.9)$ & & \\
\hline Two Days & $88(39.4)$ & $26(40.6)$ & 0.4 & $0.45(0.34-1.54)$ \\
\hline Three Days Or More & $103(46.2)$ & $24(37.5)$ & 0.15 & $1.14(0.27-1.22)$ \\
\hline \multicolumn{2}{|l|}{ Volume of pelvic drainage in $24 \mathrm{~h}$} & & \multicolumn{2}{|l|}{0.88} \\
\hline$\leq 50$ & $80(35.9)$ & $25(39.1)$ & & \\
\hline $50-100$ & $67(30)$ & $19(29.7)$ & 0.78 & $0.91(0.46-1.79)$ \\
\hline$>100$ & $76(34.1)$ & $20(31.3)$ & 0.61 & $0.84(0.43-1.64)$ \\
\hline
\end{tabular}

Values are presented as $n(\%)$;

OR, odds ratio; CI, Confidence interval;

Binary logistic regression analysis.

rocuronium (6-8 mg. $\mathrm{kg}^{-1}$ ) was administered to allow tracheal intubation, and further boluses of rocuronium were administered to maintain the train-of-four at $0-10 \%$. Anesthesia was maintained with oxygen in air $(1: 2)$, propofol, remifentanil, and sevoflurane. Morphine $\left(0.1 \mathrm{mg} \cdot \mathrm{kg}^{-1}\right)$ and palonosetron $(0.25 \mathrm{mg})$ were administered 30 minutes before the end of the operation when administration of the inhalation anesthesia was stopped. During closure of the skin, remifentanil infusion was discontinued [25], and after closure, $10 \mathrm{~mL}$ of $0.75 \%$ ropivacaine local anesthesia was infiltrated at the incision site.

All surgical procedures were performed by or under supervision of experienced surgeons according to standard protocol with laparoscopic ports $(1 \times 10 \mathrm{~mm}$ and $2-3 \times 5 \mathrm{~mm})$. The uterus was removed vaginally by volume-reducing techniques. During surgery, intra-abdominal pressure was maintained at $12-13 \mathrm{mmHg}$, and at the end of procedure, $\mathrm{CO} 2$ was evacuated by manual compression of the abdomen through the open laparoscopic ports. Intraoperative procedures and diagnosis, duration of operation, bleeding volume, and intraoperative complications were noted.

At the post anesthesia care unit (PACU), patients were titrated with morphine by the anesthesiologist as follows: Level of subjective pain intensity was obtained at 5 minutes' interval, and repeat 1-2 mg bolus dose of morphine was administered until NRS score of $<4 / 10$ was achieved, indicating adequate level of analgesia based on responses of the patients to questions by the anesthesiologist [26].

One hour later, the patients were moved to the ward; if recurrence of NRS score $\geq 4 / 10$ was observed, 50-mg suppository of indomethacin was administered though the rectum, and if the score of $\geq 4 / 10$ persisted, IM pethidine injection was administered to relieve pain [27]. The total amount of 
TA B L E 6. Multivariate analysis of risk factors of acute postoperative pain

\begin{tabular}{|c|c|c|c|c|}
\hline Risk factors & $P$ value & OR & \multicolumn{2}{|c|}{ 95\% Confidence interval } \\
\hline \multicolumn{5}{|l|}{ visceral pain } \\
\hline \multicolumn{5}{|l|}{ BMI } \\
\hline$<24$ & Reference & & & \\
\hline$\geq 24$ & 0.046 & 0.6 & 0.37 & 0.99 \\
\hline \multicolumn{5}{|c|}{ Main indication for surgery } \\
\hline Physical Findings & Reference & & & \\
\hline Descensus Uteri & 0.019 & 0.09 & 0.01 & 0.67 \\
\hline Changes In Menstruation & 0.427 & 1.26 & 0.71 & 2.25 \\
\hline Dysmenorrhea & 0.363 & 0.66 & 0.27 & 1.62 \\
\hline \multicolumn{5}{|c|}{ Post-Operative Nausea And Vomiting } \\
\hline No & Reference & & & \\
\hline Only Nausea & 0.086 & 2.05 & 0.9 & 4.65 \\
\hline Vomiting & 0.016 & 2.09 & 1.15 & 3.81 \\
\hline \multicolumn{5}{|l|}{ low back pain } \\
\hline \multicolumn{5}{|l|}{ BMI } \\
\hline$<24$ & Reference & & & \\
\hline$\geq 24$ & 0.015 & 0.51 & 0.30 & 0.88 \\
\hline \multicolumn{5}{|c|}{ Preoperative Chronic Pain } \\
\hline No & Reference & & & \\
\hline Yes & $<0.001$ & 3.61 & 2.08 & 6.24 \\
\hline \multicolumn{5}{|l|}{ The Level Of Anxiety } \\
\hline Mild & Reference & & & \\
\hline Moderate & 0.353 & 0.70 & 0.34 & 1.48 \\
\hline Severe & 0.004 & 3.46 & 1.50 & 7.98 \\
\hline \multicolumn{5}{|l|}{ The Number Of Trocar } \\
\hline Three & Reference & & & \\
\hline$\geq$ Four & 0.009 & 0.49 & 0.29 & 0.84 \\
\hline \multicolumn{5}{|l|}{ Somatic pain } \\
\hline \multicolumn{5}{|l|}{ Occupation } \\
\hline No & Reference & & & \\
\hline Yes & 0.006 & 2.28 & 1.27 & 4.09 \\
\hline \multicolumn{5}{|c|}{ A History Of Abdominal Surgery } \\
\hline No & Reference & & & \\
\hline Yes & 0.011 & 2.25 & 1.21 & 4.19 \\
\hline \multicolumn{5}{|l|}{ Pelvic Adhesions } \\
\hline No & Reference & & & \\
\hline Yes & 0.006 & 2.31 & 1.27 & 4.19 \\
\hline
\end{tabular}

Values are presented as $n(\%)$;

$O R$, odds ratio;

Multivariate logistic regression analysis.

analgesia during postoperative period (including that at PACU) and any adverse events were recorded in each patient.

Pain was assessed by the study team retrospectively on postoperative day 1 and 7 at 17:00 [28], using 11-point NRS for different types of acute postoperative pain according to the method in a previous study for average pain at rest and at movement, and worst pain [12]. For assessment of the predictors of and risk factors for intense pain scores after surgery, we considered that highest pain intensity is generally attained on postoperative day 1 , and highest pain scores are 
obtained at interview itemizing maximum pain after surgery [15]. Based on these facts, we selected maximum pain during 7 days as the measure of severe postoperative pain in the prediction analysis [23].

\subsection{Statistical analysis}

In our hospital, every year, approximately 1500 patients undergo LAVH surgery. Based on mean value of maximum acute postoperative pain after LAVH of approximately 4.5 (NRS 0$10)$, standard deviation of 2 , and permissible error $\leq 0.5$, in total, 207 patients were required, with expected loss to followup of $10 \%$.

Statistical analysis was performed with Stata 15. Baseline data are expressed as mean (SD) for continuous variables, or number of patients (\%) for binary or categorical data. Normality was determined using Kolmogorov-Smirnov tests. Box plot or scatter plot was used to detect outliers. Collinearity was examined by variance inflation factor (VIF); according to NRS score, acute postoperative pain was categorized into mild pain and moderate to severe pain [29]. Categorical data were compared using Fisher's exact test. Continuous variables were compared using Student's $t$-test (parametric) and MannWhitney U-test (nonparametric). Assumption of linearity was examined using empirical logit plots to preserve degrees of freedom. All variables with $P$-value $<0.20$ in univariate analysis were entered into multivariable logistic regression models; those with $P \leq 0.05$ by multivariable logistic regression were considered significant, and the odds ratio (OR), 95\% CIs, and $P$-value were reported. Discrimination of the model was quantified by the area under the receiver operating characteristic (ROC) curve [30]. All statistical tests were two-sided, and $P<0.05$ was considered to be statistically significant.

\section{Results}

\subsection{Study sample}

A total of 407 patients were screened, among who, 120 patients were missing complete records or without records: Forty-two (35\%) patients were excluded because of change in the mode of surgery, 38 patients did not conform to the inclusion criteria, and three patients $(2.5 \%)$ were lost to follow-up. Finally, 287 remaining patients with complete baseline data were enrolled. Flowchart of exclusion and loss to follow-up is shown in Fig. 1.

The mean [minimum-maximum] age at the time of inclusion was 49.20 [33-70] years. In the cohort of 287 patients, history of hypertension was noted in 61 patients $(21.3 \%)$, and diabetes, in only 17 patients $(5.9 \%)$; history of abdominal surgery, such as cesarean section, hysteromyoma decollement, cholecystectomy or appendectomy was noted in 74 (25.8\%) patients [31,32]. Majority of the patients were without much anxiety on preoperative day 1 , and among those, self-report of mild anxiety was obtained in $74.2 \%$ patients. In $253(88.2 \%)$ patients, birth was through vaginal route, and in 34 patients $(11.8 \%)$, it was by cesarean section (Table 1$)$.

\subsection{Preoperative pain}

In the study cohort, preoperative chronic pain was observed in 84 patients: headache in $23(8.0 \%)$ patients, cervical pain in 13 (4.5\%) female patients, low back pain in $46(16.0 \%)$ patients, shoulder pain in four $(1.4 \%)$ patients, stomachache in one patient, and pain of the limbs in five (1.7\%) female patients; in addition, dysmenorrhea was observed in 100 (34.8\%) female patients. Nevertheless, no chronic pain was reported by 129 patients before surgery.

Different types of moderate to severe pain ( $\geq 1$ type) were observed in 217 patients: one type in 116 patients, two types in 81 patients, three types in 17 patients, four types in 3 patients. Moreover, no pain or mild pain was reported in 70 patients, which suggests achievement of insufficient analgesia in our cohort.

\subsection{Postoperative pain}

Among 254 patients with postoperative visceral pain, the pain was located at the middle of the pelvic area in majority of the patients and with moderate to severe degree in 125 women. Within 2 days after surgery, low back pain was reported in 220 patients, of which 108 patients were cases of moderate to severe low back pain, and 11 patients were worst cases of severe low back pain such as spine fracture. After delivery of local anesthesia to the area of the laparoscopic ports, severe somatic pain was revealed in only six patients. Moreover, in our study, shoulder pain was observed in $44(15.3 \%)$ patients (Table 2).

In the total cohort, on postoperative day 1 , the mean NRS score of maximum postoperative visceral pain was 3.71 , and that of maximum acute postoperative low back pain was 3.17, and no significant difference was observed between the patients with visceral pain and those with low back pain; in addition, the mean NRS score of maximum acute postoperative somatic pain was 1.72 . With regard to the pain location/type, visceral pain and low back pain was observed in majority of the patients, followed by somatic pain and shoulder pain.

\subsection{Postoperative analgesia}

At PACU, analgesics were administered in 35 (12.3\%) patients, and maximum pain was 5 on NRS (0-10); particularly, on postoperative day 1, analgesics were administered in 93 $(32.4 \%)$ patients, and among those, repeat administration of analgesic agent was required in $34(36.56 \%)$ patients. After administration of pethidine, there was no recurrence of serious pain in almost all patients.

\subsection{Risk factors of acute postoperative visceral pain}

Among 24 potential risk factors included in univariate analysis, six factors were associated with acute postoperative visceral pain with statistical significance $(P<0.2)$ : medical insurance type, hypertension, diabetes, BMI, main indication for surgery, and postoperative nausea and vomiting (Table 3). All those combined were submitted to multivariate analysis; in the results, BMI, main indication for surgery, and postoperative nausea and vomiting were associated with acute postoperative 
visceral pain $(P<0.05)$ (Table 6$)$

\subsection{Risk factors of acute postoperative low back pain}

Among the risk factors of low back pain included in univariate analysis, 11 factors were associated with acute postoperative low back pain with statistical significance $(P<0.2)$ : age, medical insurance type, history of abdominal surgery, mode of delivery, BMI, main indication for surgery, preoperative chronic pain, anxiety, the number of trocar, bleeding volume, and the time of indwelling catheter (Table 4). All those combined were submitted to multivariate analysis; in the results, BMI, preoperative chronic pain, anxiety, and the number of trocar were associated with acute postoperative low back pain $(P<0.05)$ (Table 6).

\subsection{Risk factors of acute postoperative somatic pain}

In the univariate analysis, 10 factors were associated with acute postoperative somatic pain with statistical significance $(P<0.2)$ : age, occupation, hypertension, diabetes, history of abdominal surgery, mode of delivery, level of anxiety, pelvic adhesions, the number of trocar, and time of pelvic drainage (Table 5). All those combined were submitted to multivariate analysis; in the results, only occupation, pelvic adhesions, and history of abdominal surgery were associated with acute postoperative somatic pain $(P<0.05)$ (Table 6$)$.

Somatic pain was a significant factor of the length of hospital stay $(P=0.04)$, and visceral pain and somatic pain were significant factors of the increase of the time to get out of bed after surgery (each, $P<0.01$ ). Nevertheless, none of the pain types was an influencing factor of the exhaust time $(P>0.05)$.

\section{Discussion}

Many studies reported the risk factors of postoperative acute pain, but none of those focused on the different types of acute postoperative pain, which may explain the discrepancies of findings among previous studies [14, 33-35]. Our study addresses this limitation, and to the best of our knowledge, it is the first study on risk factors of postoperative acute pain according to different components of pain. Our results indicated that each of various types of acute postoperative pain have unique risk factors, which may explain the inconsistency of risk factors for postoperative pain among the previous studies.

In the LAVH operation, after laparoscopy was established, bipolar electrocoagulation was used to separate and cut off the ovarian proper ligament and the round ligament of uterus in turn. After opening the bladder peritoneal refluxing, vaginal operation was used. Main ligament of uterus, sacral ligament, and uterine artery and vein were cut off by electrocoagulation. The uterus was taken out through vagina and the vaginal stump was sutured with absorbable suture. In laparoscopic total hysterectomy (LTH), the operation is performed under the laparoscope, and there is no vaginal operation except taking out the uterus. LAVH not only broadens the indications of vaginal hysterectomy, especially for patients with pelvic adhesions, but also reduces the difficulty of operation and postopera- tive complications. This operation perfectly combines the advantages of laparoscopic surgery and vaginal hysterectomy, which greatly reduces the difficulty of operation, shortens the operation time, reduces the amount of bleeding, and is beneficial for postoperative recovery, which was worthy of clinical application.

Prospective observational study revealed the different types of acute postoperative pain; particularly, that after LAVH comprised four types, of which, visceral pain and low pain dominated over somatic pain and shoulder pain. Visceral pain and low back pain caused a major burden to the patients. Kim et al. reported inconsistencies of some areas of brain projection between visceral pain and somatic pain [36]. In our study, BMI, main indication for surgery, and postoperative nausea and vomiting were significant factors of acute postoperative visceral pain, BMI, preoperative chronic pain, anxiety, and the number of trocar were significant factors of acute postoperative low back pain, and occupation, pelvic adhesions, and history of abdominal surgery were significant factors of acute postoperative somatic pain.

In our study, $86(30 \%)$ patients reported preoperative chronic pain in $\geq 1$ areas, in agreement with data of other studies [37-39]; the majority of patients with preoperative chronic pain reported low back pain, which indicates that preoperative chronic pain was associated with an increased risk of acute low back pain. Patients who experienced chronic severe pain before the operation could easily experience adverse cognition of catastrophic pain, and these patients are usually not willing to get out of bed early; moreover, they may experience more severe postoperative low back pain [40], and preoperative chronic pain can cause central sensitization, which leads to decrease of the pain threshold and increase of the pain sensitivity $[40,41]$.

The number of trocars was significantly related to acute postoperative low back pain, which conflicts with the fact that the patients undergoing laparoscopic surgery with smaller incision experienced significantly less pain [42]. We observed that the number of trocars is a protective factor, and greater number of trocars increases the difficulty of surgery, for which patients prepare psychologically. Patients in the group with higher number of trocars achieved shorter operation time, without significance as compared to those in the group with lower number of trocars.

BMI was a protective factor against visceral pain and low back pain, which may be due to three reasons: First, obese patients have a larger proportion of subcutaneous fat, which reduces exposure of the nerve endings and consequently, reduces sensitivity to pain [43]; second, as compared with individuals with normal weight, obese patients have lower activity and workload, which supports our observation of lower incidence of joint strain before operation in the patients with obesity; third, medical staff are easily alerted when surgery is planned for obese patients, because of the challenges of the operation.

Severe preoperative anxiety was a risk factor for acute postoperative low back pain, in agreement with the findings of previous studies [23, 44]. However, only 30 patients in our study showed severe preoperative anxiety and needed sedative pills before surgery. Nevertheless, our study provides data for future study on the risk factors of different types of pain 
in patients with higher anxiety, such as those with malignant tumors undergoing laparoscopic surgery.

Surgical stress activates neuroendocrine and immunological pathways, and evokes inflammatory responses, which leads to pelvic adhesions and persistent chronic postoperative pain $[45,46]$. In our study, history of abdominal surgery $(P=$ $0.22)$ and pelvic adhesion $(P=0.49)$ were not associated with preoperative chronic pain; this result could be due to the fact that low back pain was the main component of preoperative chronic pain in the present study. However, history of abdominal surgery was significantly correlated with pelvic adhesion $(P<0.01)$, which is consistent with the results of our previous studies $[47,48]$. Both history of abdominal surgery and pelvic adhesion were associated with somatic pain (Table 6). In the assessment of the potential risk factors of different types of pain, since the factor of pelvic adhesions is associated with an increased risk of operative complications, such as injury of the bladder, bowels, and blood vessel [49], we did not delete any of those factors in the analysis.

Almost half of the patients were housewives without other occupation and they experienced more leisure than those who were employed in the workforce; during work, sitting or standing for long periods could lead to fatigue and chronic pain $[50,51]$. Our research revealed that occupation was associated with somatic pain, which supports that occupation is a risk factor of pain based on earlier reports $[52,53]$.

Anatomically, the phrenic nerve (C3-5) innervates the diaphragmatic pleural surface and connects with the supraclavicular nerve (C3-4), which relays sensory input of the acromium process, and irritation of the diaphragm leads to shoulder pain $[54,55]$. Shoulder pain after TLH may increase in intensity by intraoperative position and external compression [15]. Trendelenburg position was associated with injury of the shoulder [56]. In the present study, the risk factors of shoulder pain are not reported in the results, but the incidence of shoulder pain was lower as compared with that of previous study; 44 women with postoperative shoulder pain were included, which may influence the accuracy of our results. Study including a large population is needed to confirm the present result of the risk factors of shoulder pain.

To the best of our knowledge, this is the first study to divide acute postoperative pain into four components of visceral pain, somatic pain, shoulder pain, and low back pain, and analyze the risk factors of different types of pain, which is an important strength; the results obtained may provide foundation data for multimode analgesia and positive guidance for prevention and treatment of pain. Nevertheless, our study has some limitations. First, factors related to specific type of surgery and sub-set of population were assessed, and since the population was not global, the findings have restricted applicability. Second, only women were included, despite the fact that sex is an influencing factor of postoperative pain. In future study, different surgery types and populations with larger size should be included. Finally, the risk factors of shoulder pain are not clear, and more samples should be collected for analysis.

\section{Conclusions}

Acute postoperative visceral pain and low back pain after LAVH are important issues; future studies on visceral pain and low back pain with analgesia administration after LAVH are needed. Different types of acute pain after LAVH are correlated with preoperative, intraoperative, and postoperative factors, and each of the different types of acute postoperative pain have unique risk factors. This study found that patients with severe preoperative anxiety, preoperative chronic pain, less use of trocar or low BMI patients undergoing LAVH surgery are more likely to have moderate and severe low back pain. Clinicians should pay attention to the preoperative education of such patients, relieve preoperative anxiety and control the level of preoperative chronic pain, which is helpful to reduce postoperative acute low back pain. Moderate and severe visceral pain is easy to be found in patients with menstrual disorders, postoperative nausea and vomiting, and low BMI. Therefore, it is necessary to prevent the occurrence of postoperative nausea and vomiting, especially those with menstrual disorders. Secondly, we should analyze the causes of postoperative pain and give appropriate intervention. Only in this way can we reduce the incidence of postoperative acute pain and improve the quality of life. This finding increases present level of understanding of acute postoperative pain, and enables prediction of postoperative pain intensity and precise treatment by clinicians.

\section{ABBREVIATIONS}

BMI, body mass index; COX, cyclo-oxygenase; LAVH, laparoscope-assisted vaginal hysterectomy; LBP, low back pain; NRS, numerical rating scale; PACU, post anesthesia care unit; VAS, visual analogue scale; VIF, variance inflation factor.

\section{AUTHOR CONTRIBUTIONS}

Luping Huang, MD, and Sijia Chen designed the study, conducted the study, analyzed the data, and approved the final manuscript. Yunchang Mo and Wenwen Du designed the study and wrote the manuscript. Wenwen Du conducted the study. Yunchang Mo and Sijia Chen analyzed data and wrote and edited the manuscript. Sijia Chen and Wenwen Du contributed equally to this work and should be considered co-first authors.

\section{ETHICS APPROVAL AND CONSENT TO PARTICIPATE}

This prospective, observational cohort study was approved by the Ethics Committee (2019-030) in Clinical Research of the First Affiliated Hospital of Wenzhou Medical University and was registered at China Clinical Trial Registry: ChiCTR-ROC17013036.

\section{ACKNOWLEDGMENT}

Thank numerous individuals participated in this study. 


\section{FUNDING}

This work was supported by National Foundation of Natural Science of China (No.81803917, No.81603685 and No.81573742), Natural Science Foundation of Zhejiang Province (CN) (No. LY15H290006, No. LY19H290008) and Wenzhou Municipal Science and Technology Bureau (CN) (2018ZY003).

\section{CONFLICT OF INTEREST}

The author reports no conflicts of interest in this work.

\section{DATA AVAILABILITY}

The clinical data used to support the findings of this study are available from the corresponding author upon reasonable request.

\section{REFERENCES}

[1] McLean SA, Ulirsch JC, Slade GD, Soward AC, Swor RA, Peak DA, et al. Incidence and predictors of neck and widespread pain after motor vehicle collision among us litigants and nonlitigants. Pain. 2014; 155: 309-321.

[2] Nielsen CS, Stubhaug A, Price DD, Vassend O, Czajkowski N, Harris JR. Individual differences in pain sensitivity: genetic and environmental contributions. Pain. 2008; 136: 21-29.

[3] Coghill R, Eisenach J. Individual differences in pain sensitivity: implications for treatment decisions. Anesthesiology. 2003; 98: 13121314.

[4] Wu M, Huang K, Long C, Tsai E, Tang C. Trends in various types of surgery for hysterectomy and distribution by patient age, surgeon age, and hospital accreditation: 10-year population-based study in Taiwan. Journal of Minimally Invasive Gynecology. 2010; 17: 612-619.

[5] Allam IS, Makled AK, Gomaa IA, El Bishry GM, Bayoumy HA, Ali DF. Total laparoscopic hysterectomy, vaginal hysterectomy and total abdominal hysterectomy using electrosurgical bipolar vessel sealing technique: a randomized controlled trial. Archives of Gynecology and Obstetrics. 2015; 291: 1341-1345.

[6] Ekstein P, Szold A, Sagie B, Werbin N, Klausner JM, Weinbroum AA. Laparoscopic surgery may be associated with severe pain and high analgesia requirements in the immediate postoperative period. Annals of Surgery. 2006; 243: 41-46.

[7] Eggemann H, Ignatov A, Frauchiger-Heuer H, Amse T, Costa SD. Laparoscopic-assisted vaginal hysterectomy versus vaginal hysterectomy for benign uterine diseases: a prospective, randomized, multicenter, double-blind trial (LAVA). Archives of Gynecology and Obstetrics. 2018; 297: 479-485.

[8] Pinto PR, McIntyre T, Nogueira-Silva C, Almeida A, Araújo-Soares V. Risk factors for persistent postsurgical pain in women undergoing hysterectomy due to benign causes: a prospective predictive study. Journal of Pain. 2012; 13: 1045-1057.

[9] Choi JB, Kang K, Song MK, Seok S, Kim YH, Kim JE. Pain characteristics after total laparoscopic hysterectomy. International Journal of Medical Sciences. 2016; 13: 562-568.

[10] Ruscheweyh R, Viehoff A, Tio J, Pogatzki-Zahn EM. Psychophysical and psychological predictors of acute pain after breast surgery differ in patients with and without pre-existing chronic pain. Pain. 2017; 158 : 1030-1038.

[11] Schreiber KL, Zinboonyahgoon N, Xu X, Spivey T, King T, Dominici $\mathrm{L}$, et al. Preoperative psychosocial and psychophysical phenotypes as predictors of acute pain outcomes after breast surgery. Journal of Pain. 2019; 20: 540-556.

[12] Pinto PR, Vieira A, Pereira D, Almeida A. Predictors of acute postsurgical pain after inguinal hernioplasty. Journal of Pain. 2017; 18: 947-955.

[13] Pinto PR, McIntyre T, Ferrero R, Almeida A, Araújo-Soares V. Predictors of acute postsurgical pain and anxiety following primary total hip and knee arthroplasty. Journal of Pain. 2013; 14: 502-515.

[14] Montes A, Roca G, Sabate S, Lao JI, Navarro A, Cantillo J, et al. Genetic and clinical factors associated with chronic postsurgical pain after hernia repair, hysterectomy, and thoracotomy: a two-year multicenter cohort study. Anesthesiology. 2015; 122: 1123-1141.

[15] Choi JB, Kang K, Song MK, Seok S, Kim YH, Kim JE. Pain characteristics after total laparoscopic hysterectomy. International Journal of Medical Sciences. 2016; 13: 562-568.

[16] Tolver MA, Strandfelt P, Rosenberg J, Bisgaard T. Pain characteristics after laparoscopic inguinal hernia repair. Surgical Endoscopy. 2011; 25: 3859-3864.

[17] Tolver MA, Strandfelt P, Rosenberg J, Bisgaard T. Pain characteristics after laparoscopic inguinal hernia repair. Surgical Endoscopy. 2011; 25: 3859-3864.

[18] Krismer M, van Tulder M. Low back pain (non-specific). Best Practice \& Research Clinical Rheumatology. 2007; 21: 77-91.

[19] Gopinath B, Jagnoor J, Kifley A, Nicholas M, Blyth F, Kenardy J, et al. Differential predictors of pain severity over 12 months following noncatastrophic injury sustained in a road traffic crash. Journal of Pain. 2019; 20: 676-684

[20] Dobscha SK, Lovejoy TI, Morasco BJ, Kovas AE, Peters DM, Hart K, et al. Predictors of improvements in pain intensity in a national cohort of older veterans with chronic pain. Journal of Pain. 2016; 17: 824-835.

[21] Sipilä R, Estlander A, Tasmuth T, Kataja M, Kalso E. Development of a screening instrument for risk factors of persistent pain after breast cancer surgery. British Journal of Cancer. 2012; 107: 1459-1466.

[22] Tan K. Appropriate body-mass index for Asian populations and its implications for policy and intervention strategies. The Lancet. 2004; 363 : 157-163.

[23] Ruscheweyh R, Viehoff A, Tio J, Pogatzki-Zahn EM. Psychophysical and psychological predictors of acute pain after breast surgery differ in patients with and without pre-existing chronic pain. Pain. 2017; 158 : 1030-1038.

[24] , Jones RN, Kiely DK, Hausdorff JM, Shmerling RH, Guralnik JM, et al. Chronic musculoskeletal pain and the occurrence of falls in an older population. The Journal of the American Medical Association. 2009; 302 : 2214.

[25] Chou R, Gordon DB, Leon-Casasola OAD, Rosenberg JM, Bickler S, Brennan T, et al. Management of postoperative pain: a clinical practice guideline from the American Pain Society, the American Society of Regional Anesthesia and Pain Medicine, and the American Society of Anesthesiologists' Committee on Regional Anesthesia, Executive Commit. Journal of Pain. 2016; 17: 131-157.

[26] Gordon DB, Deleon-Casasola OA, Wu CL, Sluka K, Brennan T, Chou $\mathrm{R}$. Research gaps on practice guidelines for acute postoperative pain management in adults: findings from a review of the evidence for an American Pain Society Clinical Practice Guideline. Journal of Pain. 2015; 17: 158-166.

[27] Persson AK, Pettersson FD, Dyrehag L, Åkeson J. Prediction of postoperative pain from assessment of pain induced by venous cannulation and propofol infusion. Acta Anaesthesiologica Scandinavica. 2016; 60: 166176.

[28] Bisgaard T, Klarskov B, Rosenberg J, Kehlet H. Characteristics and prediction of early pain after laparoscopic cholecystectomy. Pain. 2001; 90: 261-269.

[29] Miaskowski C, Cooper B, Paul SM, West C, Langford D, Levine JD, et al. Identification of patient subgroups and risk factors for persistent breast pain following breast cancer surgery. Journal of Pain. 2012; 13: 11721187.

[30] Moons KGM, Kengne AP, Woodward M, Royston P, Vergouwe Y, Altman DG, et al. Risk prediction models: i. development, internal validation, and assessing the incremental value of a new (bio)marker. Heart. 2012; 98: 683-690.

[31] Lee SY, Kim CH, Kim YJ, Kim HR. Laparoscopic surgery for colorectal cancer patients who underwent previous abdominal surgery. Surgical Endoscopy. 2016; 30: 5472-5480.

[32] Kim IY, Bo RK, Kim YW. Impact of prior abdominal surgery on rates of conversion to open surgery and short-term outcomes after laparoscopic surgery for colorectal cancer. PLoS ONE. 2015; 10: e0134058. 
[33] Ghezzi F, Uccella S, Cromi A, Siesto G, Serati M, Bogani G, et al. Postoperative pain after laparoscopic and vaginal hysterectomy for benign gynecologic disease: a randomized trial. American Journal of Obstetrics and Gynecology. 2010; 203: 118.e1-118.e8.

[34] Brandsborg B, Nikolajsen L, Hansen C, Kehlet H, Jensen T. Risk factors for chronic pain after hysterectomy. Anesthesiology. 2007; 106: 10031012.

[35] Brandsborg B, Dueholm M, Nikolajsen L, Kehlet H, Jensen TS. A prospective study of risk factors for pain persisting 4 months after hysterectomy. Clinical Journal of Pain. 2009; 25: 263-268.

[36] Kim YS, Kwon SJ. High thoracic midline dorsal column myelotomy for severe visceral pain due to advanced stomach cancer. Neurosurgery. 2000; 46: 85-92.

[37] Bruce J, Thornton AJ, Scott NW, Marfizo S, Powell R, Johnston M, et al. Chronic preoperative pain and psychological robustness predict acute postoperative pain outcomes after surgery for breast cancer. British Journal of Cancer. 2012; 107: 937-946.

[38] Hetmann F, Schou-bredal I, Sandvik L, Kongsgaard UE. Does chronic pre-operative pain predict severe post-operative pain after thoracotomy? a prospective longitudinal study. Acta Anaesthesiologica Scandinavica. 2013; 57: 1065-1072.

[39] Gerbershagen HJ, Özgür E, Dagtekin O, Straub K, Hahn M, Heidenreich $\mathrm{A}$, et al. Preoperative pain as a risk factor for chronic post-surgical pain - six month follow-up after radical prostatectomy. European Journal of Pain. 2009; 13: 1054-1061.

[40] Schreiber KL, Kehlet H, Belfer I, Edwards RR. Predicting, preventing and managing persistent pain after breast cancer surgery: the importance of psychosocial factors. Pain Management. 2014; 4: 445-459.

[41] Woolf CJ. Central sensitization: implications for the diagnosis and treatment of pain. Pain. 2011; 152: S2-S15.

[42] Leggett PL, Churchman-Winn R, Miller G. Minimizing ports to improve laparoscopic cholecystectomy. Surgical Endoscopy. 2000; 14: 32-36.

[43] Kaunisto MA, Jokela R, Tallgren M, Kambur O, Tikkanen E, Tasmuth $\mathrm{T}$, et al. Pain in 1,000 women treated for breast cancer: a prospective study of pain sensitivity and postoperative pain. Anesthesiology. 2013; 119: 1410-1421.

[44] Pan PH, Tonidandel AM, Aschenbrenner CA, Houle TT, Harris LC, Eisenach JC. Predicting acute pain after cesarean delivery using three simple questions. Anesthesiology. 2013; 118: 1170-1179.

[45] Straatman J, Cuesta MA, de Lange-de Klerk ESM, van der Peet DL. Long-term survival after complications following major abdominal surgery. Journal of Gastrointestinal Surgery. 2016; 20: 1034-1041.

[46] Al-Chaer ED, Traub RJ. Biological basis of visceral pain: recent developments. Pain. 2002; 96: 221-225.

[47] van der Wal JBC, Iordens GIT, Vrijland WW, van Veen RN, Lange J, Jeekel J. Adhesion prevention during laparotomy: long-term follow-up of a randomized clinical trial. Annals of Surgery. 2011; 253: 1118-1121.

[48] Imudia A, Kumar S, Saed G, Diamond M. Pathogenesis of intraabdominal and pelvic adhesion development. Seminars in Reproductive Medicine. 2008; 26: 289-297.

[49] Molegraaf MJ, Torensma B, Lange CP, Lange JF, Jeekel J, Swank DJ. Twelve-year outcomes of laparoscopic adhesiolysis in patients with chronic abdominal pain: a randomized clinical trial. Surgery. 2017; 161 : 415-421.

[50] Lis AM, Black KM, Korn H, Nordin M. Association between sitting and occupational LBP. European Spine Journal. 2007; 16: 283-298.

[51] Hartvigsen J, Leboeufyde C, Lings S, Corder EH. Is sitting-while-at-work associated with low back pain? A systematic, critical literature review. Scandinavian Journal of Public Health. 2000; 28: 230-239.

[52] Singh S, Kumar D, Kumar S. Risk factors in cervical spondylosis. Journal of Clinical Orthopaedics and Trauma. 2014; 5: 221-226.

[53] Katz JN. Lumbar disc disorders and low-back pain: socioeconomic factors and consequences. Journal of Bone and Joint Surgery-American Volume. 2006; 88: 21-24.

[54] Bolser DC, Hobbs SF, Chandler MJ, Ammons WS, Brennan TJ, Foreman RD. Convergence of phrenic and cardiopulmonary spinal afferent information on cervical and thoracic spinothalamic tract neurons in the monkey: implications for referred pain from the diaphragm and heart. Journal of Neurophysiology. 1991; 65: 1042-1054.

[55] Zirak N, Soltani G, Hafizi L, Mashayekhi Z, Kashani I. Shoulder pain after caesarean section: comparison between general and spinal anaesthesia. Journal of Obstetrics and Gynaecology. 2012; 32: 347-349.

[56] Herron RAC. Post-operative brachial plexus paralysis. The British Medical Journal. 1952; 1: 1115-1116.

How to cite this article: Si-Jia Chen, Wen-Wen Du, Yun-Chang Mo, Jun-Lu Wang, Lu-Ping Huang. Risk factors of different types of acute pain after laparoscope-assisted vaginal hysterectomy. Signa Vitae. 2021;17(2):193-207. doi:10.22514/sv.2021.010. 